\title{
Bioethical aspects in type I neurofibromatosis
}

\author{
Codruta Diana Petchesi ${ }^{1}$, Gabriela Ciavoi ${ }^{1}$, Claudia Jurca ${ }^{1,2}$, Romana Vulturar ${ }^{3,4}$, \\ Marius Bembea ${ }^{1,2}$ \\ ${ }^{1}$ Faculty of Medicine and Pharmacy, University of Oradea, Romania \\ ${ }^{2}$ Bihor Regional Center for Medical Genetics, \\ "Dr. Gavril Curteanu" Clinical Municipal Hospital, Oradea, Romania \\ ${ }^{3}$ Department of Molecular Sciences, \\ "Iuliu Hatieganu" University of Medicine and Pharmacy, Cluj-Napoca, Romania \\ ${ }^{4}$ Cognitive Neuroscience Laboratory, Babes-Bolyai University, Cluj Napoca, Romania
}

\begin{abstract}
Type I neurofibromatosis is one of the most common monogenic disorders, being caused by abnormalities of the neurofibromin gene on chromosome 17. About half of the cases are inherited, respecting the autosomal dominant inheritance criteria, the rest are de novo cases. The clinical manifestations are multisystemic and are progressively installed, presenting inter- and intra-familial variability of clinical expression. The hereditary nature, impaired quality of life and lethal potential identify numerous and various ethical dilemmas in the diagnosis, monitoring and treatment of neurofibromatosis type 1 . Variable expressiveness and multisystemic clinical manifestations determine the unpredictable evolutionary character, associating bio-ethical dilemmas necessary to be managed in the clinical context of the disease. As a clinical applicability, we conclude that some of these problems could be avoided by informing and educating affected families about the disease, by increasing confidence in specialized services and by using molecular techniques in order to know as accurately as possible the genotype-phenotype correlation.
\end{abstract}

Keywords: neurofibromatosis, dilemmas, bioethics

\section{INTRODUCTION}

Neurofibromatosis type I (NF1 or peripheral type), MIM \# 162200 [1], is the most common form of neurofibromatosis (NF), being caused by abnormalities of the neurofibromin gene (NF1 gene) located on chromosome 17 (17q11.2). The NF1 gene regulates the production of neurofibromin, a protein involved in the regulation of cell growth. To date, more than 1,400 mutations in this gene have been identified [2]. Most gene mutations will generate the production of a dysfunctional protein, while large deletions will cause its absence. The incidence is 1: 3000 live births [3]. About half of the cases are inherited, respecting the criteria of autosomal dom- inant inheritance, the rest are de novo cases, the most plausible explanation being the high rate of mutations of the NF1 gene in germ cells. The disease has a $100 \%$ penetration (all individuals with the NF1 gene mutation have disease-specific phenotype) and large variable expressivity (clinical manifestations vary largely from one patient to another), both inter- and intrafamilial. The clinical manifestations are multisystemic and are progressively installed [4]. The major, defining features for the disease are: café-au-lait spots, axillary and inguinal freckles, peripheral neurofibromas, Lisch nodules; these features occur in most patients and are the main signs of diagnostic criteria. Occasional fea- 
tures may be: short stature, macrocephaly, neurofibromas with different visceral locations (kidney, heart, stomach, tongue, etc.), pheochromocytoma and others. The most common complications are plexiform neurofibromas, optic gliomas, skeletal dysplasias, malignization of the tumors. At the cognitive-behavioral level, the intelligence quotient (IQ) is, in most cases, average, children may have speech and learning difficulties, behavioral and personality disorders, depression and anxiety [5]. NF1 affects the quality of life through pain, limited mobility, impaired vision, breathing and sleep as well as cosmetic or aesthetic problems.

\section{CASE STUDY\#1}

Ioan, the only child in the family, was suspected from birth for the diagnosis of NF, because he had multiple café-au-lait spots and his mother was diagnosed from childhood with NF, a common form. Ioan had a normal physical and intellectual development and entered puberty at the age of 12 . Since that time, the skin pigment changes were associated with multiple subcutaneous tumors (neurofibromas). At the age of 16 , some subcutaneous neurofibromas, especially those located in the skull, degenerated malignantly, becoming voluminous and being accompanied by compression phenomena. In addition, clinical and laboratory signs were suggestive for the diagnosis of pheochromocytoma. His physical and functional condition is progressively worsening and Ioan dies at the age of 18 with to multi-organic failure and cachexia.

Since puberty, Ioan was very concerned about his suffering, always asking for information about the evolution of the disease to both doctors and his family (his mother remained inseparable from him until his death). After the age of 16, informed and aware of the malignant evolution of the disease, Ioan repeatedly asked the doctors not to inform his mother about the aggravating evolution of his disease, trying, in this way, to spare her. On the other hand, the mother also requested the doctors not to inform Ioan (still seen as a child by his mother) about the severity of the disease, due to the same desire to protect him.

The ethical dilemma raised by this case is mainly related to the communication of medical information: to whom and how medical information is transmitted in the case of teenagers, with normal intellect and aware of the disease severity. The alternatives are: exclusively to the patient; exclusively to parents; both to the patient and the parents, independently, letting each of them understand that the information is exclusive; both to the patient and the parents at the same time.

\section{CASE STUDY \#2}

Maria was the third child born into a family of four. In addition to the four births, the mother had three abortions (most likely voluntary). The first two children, as well as the fourth, showed from birth multiple hyperpigmented (café-au-lait) spots. By adolescence, all three of Maria's brothers had a normal physical and intellectual development, with no other clinical signs of illness. Their mother was diagnosed with NF1 in adolescence, her mother and sister being also affected.

Maria's birth was a shock, not only for the family but also for the doctors. In addition to the café-aulait spots (now common in the family), Maria was born with a giant left orbital tumor that monstrously deformed the facial appearance, pushing the eye slit to the base of the nasal pyramid. As a teenager, Maria has obvious complexes towards her siblings ("why she doesn't have the same moderate form of the disease?"), but mainly towards her entourage (especially her schoolmates). She is insistently concerned with the aesthetic correction of facial disfigurement and is concerned about the long-term evolution of the disease.

The case brings to our attention two dilemmas:

1. Is there the premise of a guided genetic counseling considering that the mother had a reproductive option without any restriction (7 pregnancies during 7 years)?

2. Is Maria entitled to blame her mother for not trying to prevent the disease through prenatal diagnosis and prenatal genetic testing, as the family history was known?

The long-term clinical observation of over 80 cases of NF1 in the records of the Bihor Regional Center for Medical Genetics revealed us that, sooner or later, each patient experiences a real drama related to his disease. He has a lot of uncertainties and asks himself countless questions. In our study we aimed to analyse what are the ethical issues raised by the care of these patients and which would be the solutions.

We have identified three main categories of ethical issues: problems regarding classical (clinical) and current diagnosis (genetic testing); dilemmas on life quality and social inclusion; ethical features of genetic counseling.

\section{ETHICAL ISSUES REGARDING CLASSICAL AND CURRENT DIAGNOSIS}

The classical diagnosis of NF1 is a clinical one and is made according to the criteria established by the National Institute of Health (NIH) in 1988, revised in 1997 [6,7]. The current, molecular, diagnosis is possible but rarely necessary, as it is not in- 
cluded in the protocol of patients in whom the clinical diagnosis is undoubted.

The molecular diagnosis has limited indications, being reserved for selected cases. Thus, the test may be recommended in individuals with suggestive signs which do not meet NIH criteria. Being an expensive test due to the large number of existing mutations, molecular testing is usually recommended only at the insistence of the family. A more common indication for molecular diagnosis is prenatal diagnosis if one of the parents is affected and the mutation is known. Testing is also recommended for in vitro fertilization as a preimplantation procedure. Another indication is the presence of isolated tumors in children without a family history, especially if the test may influence the treatment.

A common problem was the delayed diagnosis, due either to the ignorance of the parents or to an attitude of minimizing or even denying the existence of the disease. None of the studied cases benefited from prenatal or preimplantation diagnosis, the parents, at the time of conception, not being informed or aware of the benefits of this test. Although more than half of the studied cases were hereditary, there were affected parents who were diagnosed only in adulthood, simultaneously with the diagnosis of their affecteed child. In case of children with isolated café-au-lait spots and healthy parents, the diagnosis is often delayed, the clinical sign without functional disorders being considered irrelevant. A similar situation occurs in the case of diagnostic suspicion in a child whose parent has a mild form of the disease, motivated by the misconception that the child's phenotype will be identical to that of the parent.

Communicating the diagnosis to other family members also rises problems sometimes. Affected people and their families process all medical information through their own filter of intelligence and education. Most often, in our cases, there was a tendency to avoid the communication of the diagnosis to other family members, not even to those at risk, due to desire to protect them or simply due to ignorance or non-acceptance of the disease's severity. At the other extreme, rarely there are families which require testing of all family members, even those without the risk of inheriting the disease. Sometimes family conflicts arise due to these two different attitudes of their members.

A less common situation is the guilt of the affected parent, the fear of being held responsible for transmitting such a complex disease.

The evolution of the disease differs from patient to patient, from a benign evolution, only with caféau-lait spots, to a dramatic evolution, with the appearance of complications, sometimes even to a lethal evolution. To date, there have been few clear correlations between a particular genotype and the severity of the disease, with large scale GWAS studies (genome-wide association study) still in progress. Thus, it was observed that a wide genomic deletion of $1.4 \mathrm{Mb}$, including the NF1 gene, causes the severe form of the disease, with the development of malignant tumors of the sheath of peripheral neurons [8]. By contrast, the deletion of a single codon in exon 17 can cause a very mild phenotype. For most mutations, however, there are no clear predictions about the evolution of the disease, it is assumed that a number of modifying genes contribute to the variable phenotype of the disease. NF1 patients should be monitored periodically, annually [9], in order to detect complications as soon as possible, by a multidisciplinary team that includes geneticist, pediatrician, surgeon, dermatologist, orthopedist, ophthalmologist, ENT specialist, psychologist, neuropsychiatrist and social worker [4].

\section{ETHICAL ISSUES REGARDING LIFE QUALITY AND SOCIAL INCLUSION}

With the development of molecular genetics and the emergence of genomic medicine there was a fear that the medicalization of families with genetic diseases will occur [10]. This involves increased anxiety by anticipating the various medical problems that occur in the natural evolution of the disease and increasing the presentations to the specialist. However, in reality, each family has its own values and process the medical information through the filter of these values. In our study group, the medicalization of the family has occurred in a single family, where the mother expressed a severe form. Most patients minimize the effects or severity of the disease, presenting to the specialist only when complications occur. They do not consider that they have a chronic disorder, they continue their normal life, rarely coming for medical evaluation, usually when new signs appear. It is also the case of one of our patients who ignored the cutaneous manifestations and learning difficulties, who rarely presented for medical assessment and died at the age of 53 from cardiac arrest, caused by an existing cardiac neurofibroma.

One of the important criteria that affects the quality of life is pain, both in type and intensity. The pain occurs as a result of tumor compression, usually increasing progressively in intensity and duration, along with the growth of the tumor. There are cases where the pain becomes unbearable, reaching a stage where the patient would wish euthanasia. We found this situation in the patient from case study \# 1, who was begging for death, having an aggressive form of the disease, with malignant degenerative tumors and pheochromocytoma. 
Aesthetic discrimination is a common problem in these patients [11], being present in our patients, too. Thus, with the appearance of cutaneous neurofibromas, patients begin to be marginalized in daily social life, especially by people outside the family, who unknowingly, can invoke a possible transmissible nature of the disease. Sometimes these discriminations are made by employers and they generate complex social problems, from lack of income to lack of health insurance, respectively lack of access to medical services and depression.

In the absence of complications, the prognosis of the disease is favorable, life expectancy being close to that of the general population. The current treatment of NF1 is a symptomatic, personalized one, which addresses the prevention and treatment of complications, different from case to case, including surgical and orthopedic procedures, neurological treatment, psychological and psycho-pedagogical assistance. There are several ongoing studies regarding the treatment of plexiform tumors or inoperable tumors with protein kinase inhibitors such as Imatinib [12] and Selumetinib (Koselugo) in children over 2 years [13]. However, a serious problem is the cost of these treatments, implicitly the lack of access to treatment. One of our patients, following the development of numerous neurofibromas, including a giant plexiform one, lost her job, lost the medical insurance and no longer had access to medical services or surgical treatment.

\section{ETHICAL ISSUES REGARDING GENETIC COUNSELLING}

The reproductive decision belongs to the affected person, respectively to his family and it is recommended to be taken after a complete and correct information regarding the hereditary feature of the disorder, the evolution, prognosis and treatment of

\section{REFERENCES}

1. Online Mendelian Inheritance in man. Available at: https://www.omim. org/entry/162200.

2. Abramowicz A, Gos M. Neurofibromin in neurofibromatosis type 1- mutations in NF1 gene as a cause of disease. Dev Period Med. 2014 Jul-Sep;18(3):297-306.

3. Lyons Jones K, Crandall Jones M, Del Campo M. SMITH'S Recognizable Patterns of Human Malformation, Eighth Edition. Elsevier, 2021.

4. Rimoin DL, Connor JM, Pyeritz RE, et al. Emery and Rimoin's Principles and Practice of Medical Genetics. Edinburgh: Churchill-Livingstone, 2002.

5. Ferner RE, Thomas M, Mercer G, et al. Evaluation of quality of life in adults with neurofibromatosis 1 (NF1) using the Impact of NF1 on Quality Of Life (INF1-QOL) questionnaire. Health Qual Life Outcomes. 2017 Feb 14;15(1):34.

6. National Institutes of Health Consensus Development Conference. Neurofibromatosis: conference statement. Arch. Neurol. 1988; 45:575-578.

7. Gutmann DH, Aylsworth A, Carey JC, et al. The diagnostic evaluation and multidisciplinary management of neurofibromatosis 1 and neurofibromatosis 2. JAMA. 1997 Jul 2;278(1):51-7. the disease. In general, in the case of an individual with a positive diagnosis of NF1, regardless of the mechanism, the chances of transmitting the disease to the offspring are $50 \%$ for each pregnancy. However, the risk of recurrence in siblings is different, being variable depending on the etiopathogenetic mechanism and family history [14].

The tendency observed in our patients was to ignore the fact that there is a hereditary condition in the family, a condition with great clinical variability. Reproductive decisions were made subjectively, taking into account other criteria including, most often and erroneously, the clinical form of the affected parent (Mirabela's case). Given the great variability of the disease, patients with mild form of the disease may have offspring with severe forms and vice versa.

\section{CONCLUSIONS}

NF1 is a genetic disorder which, due to its hereditary nature, lethal potential and impairment of life, causes numerous and various ethical dilemmas in the diagnosis, monitoring and treatment of the disease. Some of these issues could be avoided by informing and educating affected families about the disease, by increasing confidence in specialized services, including the use of molecular techniques in order to know, as accurately as possible, the genotype-phenotype correlation.

\section{Acknowledgment}

All authors have equal contributions to this paper.

We mention that the names used in the case study presentations are fictitious and do not allow the identification of persons.

Conflict of interest: none declared Financial support: none declared

8. De Raedt T, Brems $\mathrm{H}$, Wolkenstein $\mathrm{P}$, et al. Elevated risk for MPNST in NF1 microdeletion patients. Am J Hum Genet. 2003 May;72(5):1288-92.

9. Fitzpatrick TB, Johnson RA, Wolff $K$, et al. Color atlas of clinical dermatology. Common and serious diseases, 3rd ed. McGraw Hill, 1997.

10. Carrieri D, Farrimond $\mathrm{H}$, Kelly $\mathrm{S}$, et al. Families dealing with the uncertainty of genetic disorders: the case of Neurofibromatosis Type 1. Sociol Health IIIn. 2016 Jun;38(5):753-67.

11. Parenti Bicudo N, de Menezes Neto BF, da Silva de Avó LR, et al. Quality of Life in Adults with Neurofibromatosis 1 in Brazil. J Genet Counsel. 2016;25:1063-74.

12. Yang F, Ingram D, Chen S, et al. Nf1-Dependent Tumors Require a Microenvironment Containing Nf1+/- and c-kit-Dependent Bone Marrow. Cell. 2008 Oct 30;135(3):437-448.

13. Gross AM, Wolters PL, Dombi E, et al. Selumetinib in Children with Inoperable Plexiform Neurofibromas. N Engl J Med. 2020 Apr 9;382(15):1430-1442.

14. Radtke HB, Sebold CD, Allison C, et al Neurofibromatosis type 1 in genetic counselling practice: recommendations of the National Society of Genetic Counselors. J Genet Couns. 2007;16(4):387-407. 